\title{
Nonseparable Radon measures and small compact spaces
}

\author{
by
}

\author{
Grzegorz P lebanek (Wrocław)
}

\begin{abstract}
We investigate the problem if every compact space $K$ carrying a Radon measure of Maharam type $\kappa$ can be continuously mapped onto the Tikhonov cube $[0,1]^{\kappa}$ ( $\kappa$ being an uncountable cardinal). We show that for $\kappa \geq \operatorname{cf}(\kappa) \geq \omega_{2}$ this holds if and only if $\kappa$ is a precaliber of measure algebras. Assuming that there is a family of $\omega_{1}$ null sets in $2^{\omega_{1}}$ such that every perfect set meets one of them, we construct a compact space showing that the answer to the above problem is "no" for $\kappa=\omega_{1}$. We also give alternative proofs of two related results due to Kunen and van Mill [18].
\end{abstract}

1. Introduction. Given a cardinal $\kappa$, denote by $\mathrm{H}(\kappa)$ the following:

Whenever $K$ is a compact space having a homogeneous Radon measure of Maharam type $\kappa$ then there is a continuous surjection from $K$ onto the Tikhonov cube $[0,1]^{\kappa}$.

We treat here only finite measures. The Maharam type of a nonatomic measure $\mu$ may be defined as the density character of the Banach space $L^{1}(\mu)$ (see [11] or [12]), and is equal to the density character of its measure algebra equipped with the Fréchet-Nikodym metric. Measures of uncountable type are often called nonseparable for obvious reasons. A measure is called homogeneous if it has the same Maharam type on every set of positive measure.

Recall that the essential part of the Maharam theorem states that if $\mu$ is a homogeneous measure of type $\kappa$ then the measure algebra of $\mu$ is isomorphic to the measure algebra of the usual product measure on $2^{\kappa}$ (equivalently, on $\left.[0,1]^{\kappa}\right)$. Thus one may formulate sentences like $\mathrm{H}(\kappa)$ in the hope of finding some topological links to Maharam's theorem.

Let us recall some basic facts and known results concerning $\mathrm{H}(\kappa)$. Let $g: K \rightarrow[0,1]^{\kappa}$ be a continuous surjection and let $\lambda_{\kappa}$ be the usual product

1991 Mathematics Subject Classification: Primary 28C15; Secondary 54A25.

Partially supported by KBN grant 2 P 30104307. 
measure on $[0,1]^{\kappa}$. The set $\Lambda$ of all Radon measures $\mu$ on $K$ such that $g(\mu)=\lambda_{\kappa}$ (i.e. $\left.\lambda_{\kappa}(B)=\mu\left(g^{-1}(B)\right)\right)$ is nonempty, convex and weak* compact so it has an extreme point, say $\mu_{0}$. Now $\mu_{0}$ is such that the spaces $L^{1}\left(\mu_{0}\right)$ and $L^{1}\left(\lambda_{\kappa}\right)$ are isometric (see Douglas [8]). It follows that the implication reverse to that in $\mathrm{H}(\kappa)$ is true for arbitrary $\kappa$.

It is well-known that a compact space $K$ admits a nonatomic Radon measure if and only if there is a continuous mapping from $K$ onto [0,1] (and this is equivalent to saying that $K$ is not scattered, [21], 19.7.6). Since $[0,1]$ can be mapped onto $[0,1]^{\omega}$, and nonatomic measures have infinite type, we see that $\mathrm{H}(\omega)$ holds true.

Haydon [14] proved that $\mathrm{H}(\kappa)$ is satisfied for every regular cardinal $\kappa$ with the property that $\tau^{\omega}<\kappa$ whenever $\tau<\kappa$. For instance, $\mathrm{H}\left(\mathfrak{c}^{+}\right)$holds. Haydon investigated $\mathrm{H}(\kappa)$ in connection with a nonseparable version of Pełczyński's theorem on Banach spaces containing $L^{1}$.

Haydon [15] and Kunen [17] presented closely related constructions which show that $\mathrm{H}\left(\omega_{1}\right)$ does not hold under the continuum hypothesis. The Kunen construction, primarily designed to give an example of a compact L-space, has been refined in various directions (see [9], [18] and Theorem 5.2 below).

What is apparently the most interesting problem concerning $\mathrm{H}(\kappa)$, is the question whether the negation of $\mathrm{H}\left(\omega_{1}\right)$ is provable within the ZFC theory. Richard Haydon conjectured that this is not the case, and that $\mathrm{H}\left(\omega_{1}\right)$ might hold under Martin's axiom and the negation of CH. All known counterexamples seem to support this conjecture.

In Section 4 of the present paper I show that, given a cardinal $\kappa \geq$ $\operatorname{cf}(\kappa) \geq \omega_{2}, \mathrm{H}(\kappa)$ holds if and only $\kappa$ is a precaliber of measure algebras (the terminology is explained in Sections 2 and 3). This covers Haydon's theorem and implies that $\mathrm{H}(\mathfrak{c})$ is undecidable in ZFC.

The next sections deal with counterexamples to $\mathrm{H}\left(\omega_{1}\right)$; I use a relatively simple method of constructing "small" compact spaces admitting a nonseparable Radon measure. I give alternative and, as I believe, simpler proofs of two results from a recent paper of Kunen and van Mill [18] (Section 5). Finally, I prove that $\mathrm{H}\left(\omega_{1}\right)$ does not hold provided the so-called weak covering number of the ideal of null subsets of $2^{\omega_{1}}$ equals $\omega_{1}$. This may indicate that the axiom " $\omega_{1}$ is a precaliber of measure algebras" does not imply $\mathrm{H}\left(\omega_{1}\right)$.

2. Preliminaries. Recall that a cardinal $\kappa$ is said to be a precaliber of a Boolean algebra $\mathbb{A}$ if for every family $\left(x_{\xi}\right)_{\xi<\kappa}$ of nonzero elements of $\mathbb{A}$ one can find a set $I \subseteq \kappa$ of power $\kappa$ such that the family $\left(x_{\xi}\right)_{\xi \in I}$ is centred, that is, $\prod_{\xi \in a} a_{\xi} \neq \mathbf{0}$ for every finite $a \subseteq I$ ([13], A2T).

It follows from the Maharam theorem that $\kappa$ is a precaliber of all measure algebras if and only if $\kappa$ is a precaliber of the measure algebra of the usual product measure on $2^{\kappa}$ (I have learned this observation from D. Fremlin). 
Let $(X, \mathcal{B}, \mu)$ be a finite measure space and let $\mathbb{A}$ be its measure algebra. For every $A \in \mathcal{B}$ we denote by $A^{*}$ the corresponding element of $\mathbb{A}$. Recall that a lifting of $\mu$ is a homomorphism $\theta: \mathbb{A} \rightarrow \mathcal{B}$ such that $\theta(a)^{\cdot}=a$ for every $a \in \mathbb{A}$ (see Section 4 of [12]). We shall need the following remark. If $\mathcal{F}$ is a family in $\mathcal{B}$ such that $F \subseteq \theta\left(F^{*}\right)$ then $\mu\left(\bigcap \mathcal{F}_{0}\right)>0$ for every finite $\mathcal{F}_{0} \subseteq \mathcal{F}$ with $\bigcap \mathcal{F}_{0} \neq \emptyset$.

Note that, given a Radon measure $\mu, \kappa$ is a precaliber of its measure algebra if and only if $\kappa$ is a caliber for the measure $\mu$ in the following sense: For every family $\left(B_{\xi}\right)_{\xi<\kappa}$ of $\mu$-measurable sets of positive measure, $\bigcap_{\xi \in X} B_{\xi} \neq \emptyset$ for some $X \subseteq \kappa$ of cardinality $\kappa$. Indeed, the latter condition is necessary, since we can replace every $B_{\xi}$ by a compact subset of positive measure; sufficiency may be checked easily by the use of lifting.

The following lemma links the notion of caliber with the covering number; it is taken from [13], A2U (and based on [6]).

Lemma 2.1. Let $(X, \Sigma, \mu)$ be a complete probability space and put $\mathcal{N}_{\mu}=$ $\{E \in \Sigma: \mu(E)=0\}$. Given a cardinal $\kappa$ of uncountable cofinality, if $\kappa$ is not a precaliber of the measure algebra of $\mu$ then there is a family $\left(E_{\xi}\right)_{\xi<\kappa} \subseteq \mathcal{N}_{\mu}$ such that $\bigcup_{\xi<\kappa} E_{\xi} \in \Sigma \backslash \mathcal{N}_{\mu}$. If , moreover, $\kappa$ is regular then the $E_{\xi}$ 's may be chosen increasing.

Now we shall recall how independent families are connected with mappings onto Tikhonov cubes (see [14] or [22]). A family $\left(\left(F_{\alpha}, H_{\alpha}\right)\right)_{\alpha<\kappa}$ is called independent if

(i) $F_{\alpha} \cap H_{\alpha}=\emptyset$ for every $\alpha<\kappa$;

(ii) $\bigcap_{\alpha \in a} F_{\alpha} \cap \bigcap_{\beta \in b} H_{\beta} \neq \emptyset$ whenever $a, b \subseteq \kappa$ are finite disjoint sets.

Lemma 2.2. A compact space $K$ admits a continuous surjection onto $[0,1]^{\kappa}$ if and only if there is an independent family $\left(\left(F_{\alpha}, H_{\alpha}\right)\right)_{\alpha<\kappa}$ such that $F_{\alpha}$ and $H_{\alpha}$ are closed subsets of $K$ for every $\alpha<\kappa$.

Let us fix some terminology and notation from topology. If $K$ is a space and $x \in K$ then $\chi(x, K)$ denotes the character (i.e. the minimal cardinality of a base at $x)$, and $\pi \chi(x, K)$ denotes the $\pi$-character of a point $x$ in $K$ (i.e. the minimal cardinality of a family $\mathcal{V}$ of nonempty open subsets of $F$ such that every neighbourhood of $x$ contains a member of $\mathcal{V}$ ).

When discussing Haydon's problem, it is worth recalling that there is a topological characterization of compact spaces admitting a surjection onto some Tikhonov cube, due to Shapirovskiu [22], Theorem 21.

THEOREM 2.3. The following are equivalent for a compact space $K$ and an infinite cardinal $\kappa$ :

(i) $K$ can be continuously mapped onto $[0,1]^{\kappa}$;

(ii) there is a closed subspace $F$ of $K$ such that $\pi \chi(x, F) \geq \kappa$ for every $x \in F$. 
We shall also need a combinatorial lemma given below. This is a corollary to the proof of the Erdős-Rado theorem on quasi-disjoint families (see [16], proof of Theorem 1.6; the well-known argument using the "pressing down lemma" gives easily the case of regular $\kappa$, see e.g. [7], Second Proof of Theorem 1.4).

LEMMA 2.4. Let $\kappa$ be a cardinal of cofinality $\geq \omega_{2}$ and let $\left(I_{\xi}\right)_{\xi<\kappa}$ be a family of countable subsets of $\kappa$. Then there are $X \subseteq \kappa$ with $|X|=\kappa$ and $R \subseteq \kappa$ with $|R|<\kappa$ such that $I_{\alpha} \cap I_{\beta} \subseteq R$ for all distinct $\alpha, \beta \in X$.

Finally, we sketch our approach to finding counterexamples to $\mathrm{H}\left(\omega_{1}\right)$ that is used in the next sections. Let $\mathcal{B}\left(2^{\omega_{1}}\right)$ be the $\sigma$-algebra of Baire sets in $2^{\omega_{1}}$ (i.e. the one generated by clopen sets), and let $\lambda_{\omega_{1}}$ denote the usual product measure on $2^{\omega_{1}}$.

We find a suitable subalgebra $\mathcal{A}$ of $\mathcal{B}\left(2^{\omega_{1}}\right)$ and define a compact space $K$ as the Stone space $\operatorname{Ult}(\mathcal{A})$ of ultrafilters (the Stone isomorphism is denoted by ${ }^{\Upsilon}$ ). Then we take the restriction of $\lambda_{\omega_{1}}$ to $\mathcal{A}$ and let $\mu$ be the unique Radon measure on $K$ defined from $\lambda_{\omega_{1}}$. Such an algebra $\mathcal{A}$ is usually obtained as the union of an increasing family of countable algebras $\mathcal{A}_{\xi}, \xi<\omega_{1}$, which are constructed inductively.

Note that in order to make $\mu$ nonseparable it suffices to make sure that for every $\xi$ there is $B \in \mathcal{A}$ such that

$$
\inf \left\{\lambda_{\omega_{1}}(A \triangle B): A \in \mathcal{A}_{\xi}\right\}>0 .
$$

If we want $K$ to be the support of $\mu$ we should ensure that $\lambda_{\omega_{1}}$ is strictly positive on $\mathcal{A}$, that is, $\lambda_{\omega_{1}}(A)>0$ for nonempty $A \in \mathcal{A}$. Note that if $\lambda_{\omega_{1}}$ is strictly positive on a countable algebra $\mathcal{A}_{\xi}$ and $B \in \mathcal{B}\left(2^{\omega_{1}}\right)$ is a set of positive measure then there is $B_{1} \subseteq B$ such that $\lambda_{\omega_{1}}$ is strictly positive on the algebra generated by $\mathcal{A}_{\xi}$ and $B_{1}$.

3. Some uncountable cardinals. In this section we fix terminology and notation concerning cardinal coefficients and formulate an auxiliary fact used in the sequel.

Let $\mathcal{J}$ be an ideal of subsets of a space $X$. Recall that the additivity $\operatorname{add}(\mathcal{J})$, the covering number $\operatorname{cov}(\mathcal{J})$ and the cofinality $\operatorname{cf}(\mathcal{J})$ of $\mathcal{J}$ are defined as

$$
\begin{aligned}
\operatorname{add}(\mathcal{J}) & =\min \{|\mathcal{E}|: \mathcal{E} \subseteq \mathcal{J}, \bigcup \mathcal{E} \notin \mathcal{J}\} \\
\operatorname{cov}(\mathcal{J}) & =\min \{|\mathcal{E}|: \mathcal{E} \subseteq \mathcal{J}, \bigcup \mathcal{E}=X\}, \\
\operatorname{cf}(\mathcal{J}) & =\min \left\{|\mathcal{E}|: \mathcal{E} \subseteq \mathcal{J}, \bigcup_{E \in \mathcal{E}} P(E)=\mathcal{J}\right\},
\end{aligned}
$$

where $P(E)$ denotes the power set of $E$. 
We shall consider two classical ideals: $\mathbb{L}$ of measure zero sets in $2^{\omega}$ and $\mathbb{K}$ of first category sets in $2^{\omega}$. Moreover, we denote by $\mathbb{L}_{\omega_{1}}$ the ideal of subsets of $2^{\omega_{1}}$ which are null with respect to the usual product measure $\lambda_{\omega_{1}}$, and by $\mathbb{C}$ the ideal generated by closed measure zero sets in $2^{\omega}$, i.e.

$$
\mathbb{C}=\left\{B \subseteq 2^{\omega}: \lambda(\bar{B})=0\right\} .
$$

Basic facts concerning ideals and their cardinal coefficients, as well as further references, may be found e.g. in [12] and [23]; see [3] and [2] for the properties of $\mathbb{C}$. It is known that the following relations between the coefficients of these ideals are always true:

$$
\omega_{1} \leq \operatorname{cov}\left(\mathbb{L}_{\omega_{1}}\right) \leq \operatorname{cov}(\mathbb{L}) \leq \operatorname{cf}(\mathbb{K})=\operatorname{cf}(\mathbb{C}) \leq \operatorname{cf}(\mathbb{L})=\operatorname{cf}\left(\mathbb{L}_{\omega_{1}}\right) \leq \mathfrak{c} .
$$

(Nothing else is provable in ZFC; see [23] for the full shape of Cichon's and related diagrams.)

Let us note that Lemma 2.1 gives the following: $\omega_{1}$ is not a caliber for the product measure on $2^{\omega_{1}}$ if and only if $\operatorname{cov}\left(\mathbb{L}_{\omega_{1}}\right)=\omega_{1}$.

The lemma given below will be used in the proof of Theorem 5.2.

Lemma 3.1. Let $\mathcal{A}$ be a countable nonatomic Boolean algebra (of sets) and let $\mu$ be a finitely additive strictly positive measure on $\mathcal{A}$.

(a) Put

$$
s(\mathcal{A})=\left\{s \in \mathcal{A}^{\omega}: s(0) \supseteq s(1) \supseteq \ldots, \lim _{n \rightarrow \infty} \mu(s(n))=0\right\} .
$$

If $\operatorname{cf}(\mathbb{K})=\omega_{1}$ then there is a family $\left(s_{\alpha}\right)_{\alpha<\omega_{1}}$ in $s(\mathcal{A})$ such that for every $t \in s(\mathcal{A})$ there is $\alpha<\omega_{1}$ such that for every $n$ and for almost all $k$ we have $t(k) \subseteq s_{\alpha}(n)$.

(b) Put

$$
p(\mathcal{A})=\left\{p \in \mathcal{A}^{\omega}: p(0) \supseteq p(1) \supseteq \ldots, \lim _{n \rightarrow \infty} \mu(p(n))>0\right\} .
$$

If $\operatorname{cf}(\mathbb{L})=\omega_{1}$ then there is a family $\left(p_{\alpha}\right)_{\alpha<\omega_{1}}$ in $p(\mathcal{A})$ such that for every decreasing sequence $t \in p(\mathcal{A})$ there is $\alpha<\omega_{1}$ such that for every $k$ and for almost all $n$ we have $t(k) \supseteq p_{\alpha}(n)$.

Pr o o f. We can assume that $\mathcal{A}$ is the algebra of clopen subsets of $2^{\omega}$ and $\mu$ is the restriction of the Lebesgue measure $\lambda$ on $2^{\omega}$.

To check (a) we may, applying the fact that $\operatorname{cf}(\mathbb{C})=\operatorname{cf}(\mathbb{K})=\omega_{1}$, take a family $\left(F_{\alpha}\right)_{\alpha<\omega_{1}}$ cofinal in $\mathbb{C}$. Write every $F_{\alpha}$ as a decreasing intersection of clopen sets $s_{\alpha}(n)$. Given $t \in s(\mathcal{A})$, the set $N=\bigcap_{k} t(k)$ is in $\mathbb{C}$, so $N \subseteq F_{\alpha}$ for some $\alpha$. For every $n$ we have $N=\bigcap_{k} t(k) \subseteq F_{\alpha} \subseteq s_{\alpha}(n)$, and thus $t(k) \subseteq s_{\alpha}(n)$ eventually holds.

We may prove (b) in a similar manner, applying the result of Cichon, Kamburelis and Pawlikowski [5]: if $\operatorname{cf}(\mathbb{L})=\omega_{1}$ then there exists a family $\left(H_{\alpha}\right)_{\alpha<\omega_{1}}$ of sets of positive measure $\lambda$ such that whenever $\lambda(B)>0$ there is $\alpha<\omega_{1}$ with $H_{\alpha} \subseteq B$. 
4. $\mathrm{H}(\kappa)$ for $\kappa \geq \omega_{2}$. We show in this section that among cardinals $\kappa$ of cofinality greater than $\omega_{1}, \mathrm{H}(\kappa)$ is fully characterized by precalibers of measure algebras.

THEOREM 4.1. Let $\kappa$ be a cardinal with $\operatorname{cf}(\kappa) \geq \omega_{2}$ and assume that $\kappa$ is a precaliber of measure algebras. Given a compact space $K$ carrying a Radon measure of Maharam type $\kappa$, there exists a continuous surjection from $K$ onto $[0,1]^{\kappa}$ (that is, $\mathrm{H}(\kappa)$ holds true).

Proof. (1) In the sequel, $2^{\kappa}$ (standing for the Cantor cube $\{0,1\}^{\kappa}$ ) is identified with the family of all subsets of $\kappa$ (thus an $x \in 2^{\kappa}$ is regarded as a subset of $\kappa$ rather than its characteristic function). A set $B \subseteq 2^{\kappa}$ depends on a set $I \subseteq \kappa$ (of coordinates) if $x \in B, y \in 2^{\kappa}$ and $x \cap I=y \cap I$ imply $y \in B$ (in other words, $B=\pi^{-1}(\pi(B))$, where $\pi$ is the natural projection onto $\left.2^{I}\right)$.

Denote by $\lambda$ the usual product measure on $2^{\kappa}$. It is well-known that $\lambda$ is inner-regular with respect to zero sets (here by a zero set in $2^{\kappa}$ we mean a closed set depending on countably many coordinates).

Let $K$ be a compact space and let $\mu$ be a Radon measure on $K$ of type $\kappa$. Since $\operatorname{cf}(\kappa) \geq \omega_{2}>\omega$, we can assume that $\mu$ is homogeneous and fix an isomorphism $\varphi: \mathbb{A}(\mu) \rightarrow \mathbb{A}(\lambda)$ between the measure algebras of $\mu$ and $\lambda$.

(2) Consider a fixed $\alpha<\kappa$. Let $V_{\alpha} \subseteq 2^{\kappa}$ be given by $V_{\alpha}=\{x \subseteq \kappa: \alpha \in$ $x\}$. Find a Borel set $A_{\alpha}$ in $K$ such that $A_{\alpha}^{\cdot}=\varphi^{-1}\left(V_{\alpha}\right)$. Next find compact sets $F_{\alpha} \subseteq A_{\alpha}$ and $H_{\alpha} \subseteq K \backslash A_{\alpha}$ such that $\mu\left(F_{\alpha}\right), \mu\left(H_{\alpha}\right) \geq 7 / 16$ (which may be done since $\mu\left(A_{\alpha}\right)=1 / 2$ and $\mu$ is a Radon measure). Now we can choose sets $B_{\alpha}$ and $C_{\alpha}$ in $2^{\kappa}$ with the properties:

(i) $B_{\alpha}$ and $C_{\alpha}$ are countable unions of zero sets;

(ii) $B_{\alpha}^{\cdot}=\varphi\left(F_{\alpha}^{\cdot}\right)$ and $C_{\alpha}^{\cdot}=\varphi\left(H_{\alpha}^{\cdot}\right)$;

(iii) $B_{\alpha} \subseteq \theta\left(\varphi\left(F_{\alpha}^{*}\right)\right)$ and $C_{\alpha} \subseteq \theta\left(\varphi\left(H_{\alpha}^{\cdot}\right)\right)$,

where $\theta$ denotes a lifting of $\lambda$.

(3) For every $\alpha<\kappa$ there is a countable set $I_{\alpha} \subseteq \kappa$ such that both $B_{\alpha}$ and $C_{\alpha}$ depend on $I_{\alpha}$. We apply Lemma 2.4 and get a set $R \subseteq \kappa$ with $|R|<\kappa$ and a set $X \subseteq \kappa$ with $|X|=\kappa$ such that $I_{\alpha} \cap I_{\beta} \subseteq R$ whenever $\alpha, \beta \in X$ and $\alpha \neq \beta$.

Denote by $\pi$ the projection from $2^{\kappa}$ onto $2^{R}$, that is, $\pi(x)=x \cap R$. To simplify the notation, we put $B_{\alpha}^{*}=\pi^{-1}\left(\pi\left(B_{\alpha}\right)\right)$ for every $\alpha$.

(4) We claim that the set $Y=\left\{\alpha \in X: \lambda\left(B_{\alpha}^{*} \cap C_{\alpha}\right)=0\right\}$ is of cardinality $<\kappa$.

Take distinct $\alpha, \beta \in Y$. Easy calculations show that $\lambda\left(B_{\alpha} \cap C_{\beta}\right) \geq 1 / 8$. Since $\lambda\left(B_{\beta}^{*} \cap C_{\beta}\right)=0$ we get

$$
\lambda\left(B_{\alpha}^{*} \triangle B_{\beta}^{*}\right) \geq \lambda\left(B_{\alpha}^{*} \backslash B_{\beta}^{*}\right) \geq \lambda\left(B_{\alpha}^{*} \cap C_{\beta}\right) \geq \lambda\left(B_{\alpha} \cap C_{\beta}\right) \geq 1 / 8 .
$$


Now, since the image measure $\lambda_{0}=\pi(\lambda)$ is of type $|R|$, and

$$
\lambda_{0}\left(\pi\left(B_{\alpha}\right) \triangle \pi\left(B_{\beta}\right)\right)=\lambda\left(B_{\alpha}^{*} \triangle B_{\beta}^{*}\right),
$$

we infer that $|Y| \leq|R|<\kappa$.

(5) We make use of the assumption that $\kappa$ is a precaliber of $\lambda$ : There is a set $Z \subseteq X \backslash Y$ with $|Z|=\kappa$ such that the family $\left(B_{\alpha}^{*} \cap C_{\alpha}\right)_{\alpha \in Z}$ is centred. We claim that the family $\left(\left(B_{\alpha}, C_{\alpha}\right)\right)_{\alpha \in Z}$ is independent.

Take any finite sets $a, b \subseteq Z$ with $a \cap b=\emptyset$. Choose $y$ so that

$$
y \in \bigcap_{\alpha \in a \cup b} B_{\alpha}^{*} \cap C_{\alpha} .
$$

For every $\alpha \in a$ we have $y \in B_{\alpha}^{*}$; thus there is $x_{\alpha} \in B_{\alpha}$ such that $x_{\alpha} \cap R=$ $y \cap R$. Defining $I(a)=\bigcup_{\alpha \in a} I_{\alpha}$ and $I(b)=\bigcup_{\beta \in b} I_{\beta}$, we put

$$
z=\bigcup_{\alpha \in a}\left(x_{\alpha} \cap I_{\alpha}\right) \cup((y \backslash R) \cap I(b)) \cup(y \cap R \backslash I(a)) .
$$

It suffices to check that

$$
z \in \bigcap_{\alpha \in a} B_{\alpha} \cap \bigcap_{\beta \in b} C_{\beta} .
$$

For any $\gamma \in a$ we have $I_{\gamma} \cap I(b) \subseteq R$ and thus

$$
z \cap I_{\gamma}=\bigcup_{\alpha \in a}\left(x_{\alpha} \cap I_{\alpha} \cap I_{\gamma}\right)=\left(x_{\gamma} \cap I_{\gamma}\right) \cup \bigcup_{\alpha \in a \backslash\{\gamma\}}\left(x_{\alpha} \cap I_{\alpha} \cap I_{\gamma}\right)=x_{\gamma} \cap I_{\gamma} .
$$

Since $x_{\gamma} \in B_{\gamma}$ and $B_{\gamma}$ depends on the set $I_{\gamma}$, we get $z \in B_{\gamma}$.

Now take any $\gamma \in b$. Then for every $\alpha \in a$ we have $x_{\alpha} \cap I_{\alpha} \cap I_{\gamma}=y \cap I_{\alpha} \cap I_{\gamma}$ and hence

$$
\begin{aligned}
z \cap I_{\gamma} & =\bigcup_{\alpha \in a}\left(x_{\alpha} \cap I_{\alpha} \cap I_{\gamma}\right) \cup\left((y \backslash R) \cap I(b) \cap I_{\gamma}\right) \cup\left(y \cap R \cap I_{\gamma} \backslash I(a)\right) \\
& =\left(y \cap I_{\gamma} \cap I(a)\right) \cup\left((y \backslash R) \cap I_{\gamma}\right) \cup\left(y \cap R \cap I_{\gamma} \backslash I(a)\right)=y \cap I_{\gamma} .
\end{aligned}
$$

Since $y \in C_{\gamma}$ and $C_{\gamma}$ depends on $I_{\gamma}$ we get $z \in C_{\gamma}$, and the claim is verified.

(6) Now (i)-(ii) of (2), (5) and the remark from Section 2 imply that in fact we have

$$
\lambda\left(\bigcap_{\alpha \in a} B_{\alpha} \cap \bigcap_{\beta \in b} C_{\beta}\right)>0
$$

whenever $a, b$ are disjoint finite sets in $Z$. This implies immediately that the family $\left(\left(F_{\alpha}, H_{\alpha}\right)\right)_{\alpha \in Z}$ is independent. We apply Lemma 2.2 and the proof is complete.

Part (a) of the next theorem was proved in [20] for successor $\kappa$ by a more complicated argument.

THEOREM 4.2. (a) If $\kappa$ is a cardinal with $\mathrm{cf}(\kappa) \geq \omega_{2}$ such that $\kappa$ is not a caliber for the measure $\lambda_{\kappa}$ then $\mathrm{H}(\kappa)$ does not hold. 
(b) If , moreover, $\kappa$ is a regular cardinal and there is $\tau<\kappa$ such that $\kappa$ is not a caliber for the measure $\lambda_{\tau}$ on $2^{\tau}$, then there is a compact space $K$ admitting a Radon measure of type $\kappa$ and such that $\chi(x, K)<\kappa$ for every $x \in K$.

Proof. (a) Choose a family $\left(C_{\xi}\right)_{\xi<\kappa}$ of compact subsets of $2^{\kappa}$ of positive measure witnessing that $\kappa$ is not a caliber for $\lambda_{\kappa}$. Without difficulty we may find compact sets $F_{\xi}$ such that $F_{\xi} \subseteq C_{\xi}$ and

$$
\inf \left\{\lambda_{\kappa}\left(A \triangle F_{\xi}\right): A \in \mathcal{A}_{\xi}\right\}>0,
$$

where $\mathcal{A}_{\xi}$ is the algebra generated by the family $\left\{F_{\alpha}: \alpha<\xi\right\}$. We shall check that the Stone space $K$ of the algebra $\mathcal{A}=\bigcup_{\xi<\kappa} \mathcal{A}_{\xi}$ is the required space. It is clear that there is a Radon measure of type $\kappa$ on $K$.

Given an arbitrary closed subset $H$ of $K$, we take a maximal subfamily $\mathcal{F}_{0}$ of $\mathcal{F}=\left\{F_{\xi}: \xi<\kappa\right\}$ for which $\mathcal{H}=\left\{\widehat{F} \cap H: F \in \mathcal{F}_{0}\right\}$ is centred. It follows that $\bigcap \mathcal{H}$ consists of a single point of $H$, say $x$. Now $\chi(x, H)<\kappa$ since $\left|\mathcal{F}_{0}\right|<\kappa$ and finite intersections of elements from $\mathcal{H}$ form a base at $x$. It follows from Theorem 2.3 that $K$ cannot be continuously mapped onto $[0,1]^{\kappa}$ and hence $K$ is a counterexample to $\mathrm{H}(\kappa)$.

(b) By the assumption and Lemma 2.1 there is an increasing family $\left(N_{\xi}\right)_{\xi<\kappa}$ of $\lambda_{\tau}$-null sets in $2^{\tau}$ with $\bigcup_{\xi<\kappa} N_{\xi}=2^{\tau}$. For every $\xi$ choose an open set $V_{\xi} \supseteq N_{\xi}$ with $\lambda_{\tau}\left(V_{\xi}\right)<1 / 2$.

Denote by $\pi: 2^{\kappa} \rightarrow 2^{\tau}$ the natural projection onto the first $\tau$ coordinates. Put $U_{\xi}=\pi^{-1}\left(V_{\xi}\right)$ and let $\mathcal{A}_{0}$ be the algebra of clopen subsets of $2^{\kappa}$ depending on the first $\tau$ coordinates.

Now we choose compact sets $F_{\xi}$ such that (**) is satisfied and $F_{\xi} \subseteq$ $2^{\kappa} \backslash U_{\xi}$ for every $\xi$. Taking $K$ as above, we check that the character of points of $K$ is less than $\kappa$.

Given $x \in K$, put $C=\bigcap\left\{A \in \mathcal{A}_{0}: A \in x\right\}$. Then $\pi(C)=\{t\}$ for some $t \in 2^{\tau}$. Therefore there is $\alpha<\kappa$ such that $t \in N_{\xi} \subseteq V_{\xi}$ for $\xi \geq \alpha$. Consequently, for every $\xi \geq \alpha$ there is $A \in \mathcal{A}_{0}$ with $A \in x$ and $A \cap F_{\xi}=\emptyset$. It follows that the algebra generated by $\mathcal{A}_{0}$ and $\left\{F_{\beta}: \beta<\alpha\right\}$ contains a base at $x$. Thus $\chi(x, K)<\kappa$ and the proof is complete.

Corollary 4.3. Given $\kappa$ with $\operatorname{cf}(\kappa) \geq \omega_{2}, \mathrm{H}(\kappa)$ is equivalent to the fact that $\kappa$ is a precaliber of measure algebras.

If a regular cardinal $\kappa$ satisfies $\tau^{\omega}<\kappa$ whenever $\tau<\kappa$ then $\kappa$ is a precaliber of every ccc space (see 5.2 of [7]), so $\kappa$ is a precaliber of every measure algebra. Thus Theorem 4.1 covers Haydon's result mentioned in the introduction.

Note that if $\kappa=\operatorname{add}(\mathbb{L})=\operatorname{cov}(\mathbb{L})$ then $\kappa$ is not a precaliber of the ordinary measure algebra, and thus $\mathrm{H}(\kappa)$ is not true. In particular, assuming $\mathfrak{c}=\operatorname{add}(\mathbb{L})$ we have non $\mathrm{H}(\mathfrak{c})$. 
Now let $\lambda$ be the product measure on $2^{\mathfrak{c}}$ and let $\mathcal{N}$ be the ideal of $\lambda$-negliglible sets. Assume that $\mathfrak{c}=\omega_{2}$ and that $\lambda^{*}(D)=1$ for some set $D \subseteq 2^{\mathfrak{c}}$ with $|D|=\omega_{1}$. Then $\mathfrak{c}$ is a precaliber of the measure algebra of $\lambda$. Indeed, otherwise there is an increasing family $\left(N_{\alpha}\right)_{\alpha<\mathfrak{c}}$ in $\mathcal{N}$ such that $\bigcup_{\alpha<\mathfrak{c}} N_{\alpha}=2^{\mathfrak{c}}$ (see Lemma 2.1). But this implies $D \subseteq N_{\alpha}$ for some $\alpha<\mathfrak{c}$, a contradiction.

The above remarks and Corollary 4.3 show that $\mathrm{H}(\mathfrak{c})$ is relatively consistent with and independent of the usual axioms.

5. Some counterexamples to $\mathrm{H}\left(\omega_{1}\right)$. There are several natural classes of compact spaces that cannot be mapped onto $[0,1]^{\omega_{1}}$ (first-countable, sequential, with countable tightness etc.). Given such a class $\mathcal{C}$ of compact spaces, one may ask if $\mathrm{H}\left(\omega_{1}\right)$ is true whenever $K \in \mathcal{C}$, which amounts to asking whether every Radon measure defined on some $K \in \mathcal{C}$ is separable. Such particular problems have been solved for the class of first-countable spaces and Corson compacta (see [18]-[20]).

Recall that a compact space $K$ is said to be Corson compact if $K$ can be embedded, for some $\kappa$, into the subset of $\mathbb{R}^{\kappa}$ consisting of elements with countable support (see [1] for properties of Corson compacta and further references). For our purpose it is sufficient to recall that, according to Rosenthal's theorem, a compact zero-dimensional space $K$ is Corson compact if and only if there exists a point-countable family $\mathcal{D}$ of clopen subsets of $K$ such that $\mathcal{D}$ separates points of $K$ (point-countability means $|\{D \in \mathcal{D}: x \in D\}| \leq \omega$ for every $x \in K)$.

It follows from Theorem 2.3 (or may be checked directly) that no Corson compactum and no first-countable space can be mapped continuously onto $[0,1]^{\omega_{1}}$. Thus any of such spaces carrying a nonseparable Radon measure witnesses that $\mathrm{H}\left(\omega_{1}\right)$ does not hold. Assuming $\operatorname{cov}\left(\mathbb{L}_{\omega_{1}}\right)=\omega_{1}$, Kunen and van Mill [18] constructed a first-countable Corson compact space $K$ with a nonseparable measure $\mu$. Moreover, under $\operatorname{cf}(\mathbb{L})=\omega_{1}$, such $K$ and $\mu$ may have other interesting properties. On the other hand, I showed in [20] that, assuming $\operatorname{cov}\left(\mathbb{L}_{\omega_{1}}\right)>\omega_{1}$, that is, if $\omega_{1}$ is a precaliber of measure algebras, every Radon measure on a first-countable space is separable.

Another class that may be considered here is that of compact spaces of countable tightness. Recall that $K$ has a countable tightness if for every $A \subseteq K$ and $x \in \bar{A}$ there is a countable set $I \subseteq A$ with $x \in \bar{I}$. Since countable tightness implies countable $\pi$-character hereditarily, no countably tight compact space can be mapped onto $[0,1]^{\omega_{1}}$ (see [22]). It is an open question whether Radon measures on countably tight spaces are separable provided $\omega_{1}$ is a precaliber of measure algebras.

The theorem below has been obtained by Kunen and van Mill [18]. 
THEOREM 5.1. If $\operatorname{cov}\left(\mathbb{L}_{\omega_{1}}\right)=\omega_{1}$ then there exists a Corson compact first-countable space that supports a nonseparable Radon measure.

Proof. Choose an increasing family $\left(N_{\xi}\right)_{\xi<\omega_{1}} \subseteq \mathbb{L}_{\omega_{1}}$ that covers $2^{\omega_{1}}$. We construct inductively compact sets $F_{\xi, n} \subseteq 2^{\omega_{1}}$ with the properties:

(i) $F_{\xi, n} \subseteq 2^{\omega_{1}} \backslash N_{\xi}$ for every $\xi$ and $n$;

(ii) $F_{\xi, n} \subseteq F_{\xi, n+1}$ and $\lambda_{\omega_{1}}\left(\bigcup_{n \in \omega} F_{\xi, n}\right)=1$ for every $\xi<\omega_{1}$;

(iii) given $\beta<\alpha<\omega_{1}$, for every $n$ there is $k$ such that $F_{\alpha, n} \subseteq F_{\beta, k}$;

(iv) $F_{\xi, 0}$ witnesses $(*)$ from Section 2 , where $\mathcal{A}_{\xi}$ is the algebra generated by all $F_{\beta, n}, \beta<\xi, n \in \omega$.

The construction is straightforward (for the limit cardinal $\xi$ choose an increasing sequence $\xi_{i}$ that is cofinal in $\xi$ and note that for every $\delta>0$ there is $\varphi \in \omega^{\omega}$ with $\left.\lambda_{\omega_{1}}\left(\bigcap_{i} F_{\xi_{i}, \varphi(i)}\right)>1-\delta\right)$.

Let $\mathcal{F}$ be the family of all $F_{\xi, n}$ 's, put $\mathcal{A}=\bigcup_{\xi<\omega_{1}} \mathcal{A}_{\xi}$ and consider the space $K=\operatorname{Ult}(\mathcal{A})$. It follows from compactness and (i) that $\mathcal{F}$ is pointcountable. Hence $\{\widehat{F}: F \in \mathcal{F}\}$ is a point-countable separating family and so $K$ is Corson compact.

Given $x \in K$, the family $\{F \in \mathcal{F}: F \in x\}$ is countable. Therefore, there is $\alpha<\omega_{1}$ such that $F_{\alpha, n} \notin x$ for every $n$. Now (iii) implies that

$$
\left\{A \in \mathcal{A}_{\alpha}: A \in x\right\} \cup\left\{2^{\omega_{1}} \backslash F_{\alpha, n}: n \in \omega\right\},
$$

gives a base at $x$. Thus $K$ is first-countable. Now, letting $L$ be the support of $\mu$, we infer that $L$ is Corson compact and first-countable, so the proof is complete.

For the sake of the next theorem recall that an $L$-space is a nonseparable topological space that is hereditarily Lindelöf (every family of its open subsets has a countable subfamily with the same union). Part (b) of the theorem below is due to Kunen and van Mill [18]. The idea of using a normal Radon measure which can recognize metrizable subsets in a construction of an L-space appeared already in Kunen [17] (normality of a Radon measure means that sets of positive measure have nonempty interior). Part (a) needs a weaker assumption, but we do not know whether a space as in (a) is hereditarily Lindelöf.

TheOrem 5.2. (a) If $\operatorname{cf}(\mathbb{K})=\omega_{1}$ then there is a Corson compact space $K$ with a nonseparable measure $\mu$ such that a closed set $H \subseteq K$ is metrizable if and only if $\mu(H)=0$.

(b) If $\operatorname{cf}(\mathbb{L})=\omega_{1}$ then there is a Corson compact space $K$ with a Radon measure $\mu$ and

(1) $\mu$ is a nonseparable normal measure on $K$;

(2) $\mu(N)=0$ if and only if $N$ is metrizable, for arbitrary $N$;

(3) $K$ is a Corson compact L-space. 
Proof. (a) We construct an increasing sequence $\left(\mathcal{A}_{\alpha}\right)_{\alpha<\omega_{1}}$ of countable subalgebras of $\mathcal{B}\left(2^{\omega_{1}}\right)$, and, for every $\alpha$, denote by $\left(s_{\beta}^{\alpha}\right)_{\beta<\omega_{1}} \subseteq s\left(\mathcal{A}_{\alpha}\right)$ families of sequences as in Lemma 3.1(a) (we keep the notation of that lemma).

We start by letting $\mathcal{A}_{0}$ be the algebra of clopen sets in $2^{\omega_{1}}$ depending on the first $\omega$ coordinates. At step $\xi$ we find a set $B$ with $\lambda_{\omega_{1}}(B)>0$ such that whenever $\alpha, \beta<\xi$ then there is $n \in \omega$ with $s_{\beta}^{\alpha}(n) \cap B=\emptyset$ (since we only have to omit countably many sequences on which the measure tends to zero, this may be done easily). Next we find a set $F_{\xi} \subseteq \bar{F}_{\xi} \subseteq B$ such that

$$
\inf \left\{\lambda_{\omega_{1}}\left(A \triangle F_{\xi}\right): A \in \mathcal{A}_{\xi}\right\}>0,
$$

and define $\mathcal{A}_{\xi+1}$ to be the algebra generated by $\mathcal{A}_{\xi}$ and $F_{\xi}$. Using the remark from Section 2 we can have $\lambda_{\omega_{1}}$ strictly positive on every $\mathcal{A}_{\xi}$. Finally, letting $\mathcal{A}=\bigcup_{\xi<\omega_{1}} \mathcal{A}_{\xi}$, we take $K$ to be the Stone space of $\mathcal{A}$. Clearly $\widehat{\mathcal{A}}_{0} \cup\left\{\widehat{F}_{\xi}\right.$ : $\left.\xi<\omega_{1}\right\}$ is a point-countable separating family so $K$ is Corson compact.

For a given compact $H \subseteq K$ of measure zero there is a decreasing sequence of clopen sets $\left(\widehat{A}_{k}\right)_{k \in \omega}$ such that $H \subseteq \bigcap_{k \in \omega} \widehat{A}_{k}$ and $\lambda_{\omega_{1}}\left(A_{k}\right) \rightarrow 0$. Thus $t=\left(A_{k}\right) \in s\left(\mathcal{A}_{\alpha}\right)$ for some $\alpha<\omega_{1}$. Now $t$ is eventually dominated by some $s_{\beta}^{\alpha}$ as in Lemma 3.1(a). Consequently, $\mathcal{A}_{\xi}$ where $\xi=\max (\alpha, \beta)$ gives a topological base for $H$. Indeed, for $\eta \geq \xi$ we have $F_{\eta} \cap s_{\beta}^{\alpha}(n)=\emptyset$ for large $n$ so there is $k$ such that $A_{k} \cap B_{\eta}=\emptyset$; thus $\widehat{B}_{\eta} \cap H=\emptyset$.

It may happen that there is a compact metric $H$ with $\mu(H)>0$. Now it suffices, however, to take a maximal (necessarily countable) family $\mathcal{H}$ of pairwise disjoint such sets and, since $\mu$ is nonseparable, find a compact set $L \subseteq K \backslash \bigcup \mathcal{H}$ of positive measure, and the proof of (a) is complete.

(b) To prove (b) we carry out the same construction as above, complemented as follows.

For every algebra $\mathcal{A}_{\xi}$ we denote by $\left(t_{\beta}^{\alpha}\right)_{\beta<\omega_{1}} \subseteq p\left(\mathcal{A}_{\xi}\right)$ a family as in Lemma 3.1(b). Given the algebra $\mathcal{A}_{\xi}$, for every $\eta, \zeta<\xi$ we find a set $F_{\zeta}^{\eta}$ of positive measure with $F_{\zeta}^{\eta} \subseteq \overline{F_{\zeta}^{\eta}} \subseteq \bigcap_{n \in \omega} t_{\zeta}^{\eta}$ such that for every $\alpha, \beta<\xi$ the sequence $s_{\beta}^{\alpha}$ is eventually disjoint from $F_{\zeta}^{\eta}$. Now we let $\mathcal{A}_{\xi+1}$ be the algebra generated by $\mathcal{A}_{\xi}, F_{\xi}$ and $\left\{F_{\zeta}^{\eta}: \eta, \zeta<\xi\right\}$.

This modification makes $\mu$ normal. In fact, suppose that $X \subseteq K$ has an empty interior but $\mu(X)>0$. We may assume that $X$ is closed; since $K$ is a ccc space there is a compact $\mathcal{G}_{\delta}$ set $Z \supseteq X$ with empty interior. There is $\xi<\omega_{1}$ and a decreasing sequence $\left(A_{k}\right)_{k \in \omega} \subseteq \mathcal{A}_{\xi}$ with $Z=\bigcap_{k \in \omega} \widehat{A}_{k}$. Now there is $\eta$ such that for every $k$ and for almost all $n$ we have $A_{k} \supseteq p_{\eta}^{\xi}(n)$. It follows that $F_{\eta}^{\xi} \subseteq A_{k}$ so $Z$ has a nonempty interior, a contradiction.

(2) is satisfied, for if $\mu(N)=0$ then $\mu(\bar{N})=0$ by normality, and $\bar{N}$ is metrizable (which may be checked as in (a)).

The fact that $K$ is an L-space now follows easily (as in [18]). Indeed, $K$ cannot be separable since a separable Corson compactum is metrizable. 
Given any family $\mathcal{V}$ of open subsets of $K$, there is a countable subfamily $\mathcal{V}_{0}$ with $\mu(E)=0$, where $E=\bigcup \mathcal{V} \backslash \bigcup \mathcal{V}_{0}$. Since $E$ is of measure zero, it is metrizable and thus is covered by another countable subfamily $\mathcal{V}_{1}$. Now $\mathcal{V}_{0} \cup \mathcal{V}_{1}$ covers $\cup \mathcal{V}$ and we are done.

6. $\mathrm{H}\left(\omega_{1}\right)$ and weak coverings. Brendle, Judah and Shelah [4] considered another cardinal invariant of the ideal $\mathbb{L}$ that is relevant here. The weak covering $\operatorname{wcov}(\mathbb{L})$ is the minimal cardinality of a family $\mathcal{E} \subseteq \mathbb{L}$ such that $2^{\omega} \backslash \bigcup \mathcal{E}$ does not contain a perfect set. Weak covering is also discussed in [2], where it is denoted by $\operatorname{cov}^{P}$. Clearly one has

$$
\operatorname{add}(\mathbb{L}) \leq \operatorname{wcov}(\mathbb{L}) \leq \operatorname{cov}(\mathbb{L}) .
$$

It is known that both $\operatorname{wcov}(\mathbb{L})<\operatorname{cov}(\mathbb{L})$ and $\operatorname{wcov}(\mathbb{L})=\operatorname{cov}(\mathbb{L})$ are relatively consistent (see [2], Theorems 3.2.17 and 2.5.14). It is shown in [4] that $\operatorname{wcov}(\mathbb{L}) \leq \max (\mathfrak{b}, \operatorname{non}(\mathbb{L}))$.

Let $\mu$ be a nonatomic Radon measure $\mu$ defined on a topological space $K$. We shall always write $\mathcal{N}_{\mu}$ for the ideal of $\mu$-null sets. One may consider the weak covering of $\mathcal{N}_{\mu}$ defined analogously:

$$
\operatorname{wcov}\left(\mathcal{N}_{\mu}\right)=\min \left\{|\mathcal{E}|: \mathcal{E} \subseteq \mathcal{N}_{\mu}, K \backslash \bigcup \mathcal{E} \text { contains no perfect set }\right\},
$$

where "perfect" means "nonempty closed without isolated points".

In particular, we can consider $\operatorname{wcov}\left(\mathbb{L}_{\omega_{1}}\right)$. Note that $\operatorname{wcov}\left(\mathbb{L}_{\omega_{1}}\right) \leq$ $\operatorname{wcov}(\mathbb{L})$. Indeed, put $\kappa=\operatorname{wcov}(\mathbb{L})$; for every $\alpha<\omega_{1}$ let $\left(N_{\xi}^{\alpha}\right)_{\xi<\kappa}$ be a family of null sets in $2^{\alpha}$ whose union meets every perfect subset of $2^{\alpha}$. Now the family $\left\{\pi_{\alpha}^{-1}\left(N_{\xi}^{\alpha}\right): \alpha<\omega_{1}, \xi<\kappa\right\}$, where $\pi_{\alpha}: 2^{\omega_{1}} \rightarrow 2^{\alpha}$ is the natural projection, meets every perfect subset of $2^{\omega_{1}}$.

Let us recall elementary facts related to perfectness. Say that $\left(D_{s}\right)_{s \in 2<\omega}$ is a dyadic system (in a space $K$ ) if $D_{s}$ is nonempty and closed, $D_{s i} \subseteq D_{s}$, and $D_{s 0} \cap D_{s 1}=\emptyset$ for every $s \in 2^{<\omega}$ and $i \in\{0,1\}$. Here $2^{<\omega}=\bigcup_{n \in \omega} 2^{n}$; if $s \in 2^{n}$ and $i \in\{0,1\}$ then $s i \in 2^{n+1}$ is an extension of $s$.

Lemma 6.1. Let $K$ be a compact space and let $F \subseteq K$ be its closed subset.

(a) If $F$ can be continuously mapped onto a perfect set then $F$ contains a perfect set.

(b) If there is a dyadic system $\left(D_{s}\right)_{s \in 2<w}$ in $K$ with $D_{s} \cap F \neq \emptyset$ for every $s \in 2^{<\omega}$ then $F$ contains a perfect set.

Proof. If $g$ is a continuous surjection from $F$ onto a perfect set $P$ then $g$ is irreducible on some closed $F_{0} \subseteq F$, so $F_{0}$ is perfect.

To check (b) put $H=F \cap \bigcap_{n \in \omega} \bigcup_{s \in 2^{n}} D_{s}$. Given $t \in 2^{\omega}$, let $g(x)=t$ for $x \in H \cap \bigcap_{n \in \omega} D_{t \mid n}$. This defines a continuous mapping from $H$ onto $2^{\omega}$, so $H$ contains a perfect set by (a). 
The results presented below show that weak coverings are closely related to the existence of nonseparable Radon measures on spaces having a lot of points of countable character.

THEOREM 6.2. If $\operatorname{wcov}\left(\mathbb{L}_{\omega_{1}}\right)=\omega_{1}$ then there exists a compact space $K$ having a nonseparable Radon measure, and such that for every perfect $P \subseteq K$ there is $x \in P$ with $\chi(x, K)=\omega$ (in particular, $\mathrm{H}\left(\omega_{1}\right)$ does not hold).

Proof. We adapt here the argument used in the proof of Theorem 5.1.

Choose an increasing family $\left(N_{\xi}\right)_{\xi<\omega_{1}} \subseteq \mathbb{L}_{\omega_{1}}$ whose union meets every perfect set in $2^{\omega_{1}}$. We construct inductively compact sets $F_{\xi, n} \subseteq 2^{\omega_{1}}$ with the properties:

(i) $F_{\xi, n} \subseteq 2^{\omega_{1}} \backslash N_{\xi}$ for every $\xi$ and $n$;

(ii) $F_{\xi, n} \cap F_{\xi, k}=\emptyset$ if $n \neq k$, and $\lambda_{\omega_{1}}\left(\bigcup_{n \in \omega} F_{\xi, n}\right)=1$ for every $\xi<\omega_{1}$;

(iii) given $\beta<\alpha<\omega_{1}$, for every $n$ there is $k$ such that $F_{\alpha, n} \subseteq \bigcup_{i<k} F_{\beta, i}$;

(iv) $F_{\xi, 0}$ witnesses $(*)$ from Section 2 , where $\mathcal{A}_{\xi}$ is the algebra generated by all $F_{\beta, n}, \beta<\xi, n \in \omega$.

We again consider the family $\mathcal{F}$ of all $F_{\xi, n}$ 's, the algebra $\mathcal{A}$ generated by $\mathcal{F}$ and the space $K=\operatorname{Ult}(\mathcal{A})$. Let $H$ be a perfect subset of $K$; we are to find an element of $H$ of countable character.

We claim that there is $\xi<\omega_{1}$ such that $H_{0}=H \backslash \bigcup_{n \in \omega} \widehat{F}_{\xi, n} \neq \emptyset$. If this is so, every $x \in H_{0}$ has a local base contained in $\mathcal{A}_{\xi+1}$ in view of (iii). Thus the proof will be complete if we verify the claim.

Suppose otherwise; then $H \subseteq \bigcup_{n \in a(\xi)} \widehat{F}_{\xi, n}$ for every $\xi<\omega_{1}$, where the (necessarily finite) set $a(\xi)$ is defined by $a(\xi)=\left\{n \in \omega: \widehat{F}_{\xi, n} \cap H \neq \emptyset\right\}$. Let

$$
P=\bigcap_{\xi<\omega_{1}} \bigcup_{n \in a(\xi)} F_{\xi, n} .
$$

Given $t \in P$, for every $\xi$ there is $\varphi(\xi) \in \omega$ such that $t \in F_{\xi, \varphi(\xi)}$. Note that $\bigcap_{\xi<\omega_{1}} \widehat{F}_{\xi, \varphi(\xi)}$ consists of a single point, say $x$, with $x \in H$. We put $g(t)=x$.

In this way we have defined a surjection from $P$ onto $H$ which is easily seen to be continuous. Hence $P$ contains a perfect set. On the other hand, $P \cap N_{\xi}=\emptyset$ for every $\xi$, and this is a contradiction.

It is very likely that $\operatorname{wcov}\left(\mathbb{L}_{\omega_{1}}\right)<\operatorname{cov}\left(\mathbb{L}_{\omega_{1}}\right)$ is relatively consistent. If this is the case then Theorem 6.2 shows that $\mathrm{H}\left(\omega_{1}\right)$ is not implied by the axiom " $\omega_{1}$ is a precaliber of measure algebras".

Added in proof. David Fremlin sent me the following remark due to Max Burke: Adding $\omega_{2}$ random reals to a model of $\mathrm{CH}$ we have $\operatorname{cov}\left(\mathbb{L}_{\omega_{1}}\right)=\omega_{2}$ but $\operatorname{wcov}(\mathbb{L})=\omega_{1}$ and hence $\operatorname{wcov}\left(\mathbb{L}_{\omega_{1}}\right)=\omega_{1}$. So this is a model in which $\omega_{1}$ is a precaliber of measure algebras but $\mathrm{H}\left(\omega_{1}\right)$ is false.

The next result offers a partial converse to the theorem above. It is proved by adapting an idea from [20]. 
TheOREM 6.3. Suppose that $K$ is a compact space such that for every perfect subset $P$ of $K$ there is $x \in P$ with $\chi(x, K)=\omega$, and admitting a nonseparable Radon measure. Then there exists a Radon measure $\mu$ on $K$ such that $\operatorname{wcov}\left(\mathcal{N}_{\mu}\right)=\omega_{1}$.

Proof. Since $K$ carries a nonseparable Radon measure, it follows that there exists a homogeneous Radon measure $\mu$ on $K$ of Maharam type $\omega_{1}$ (see [20], Lemma 2 or [14], Proposition 2.1). We shall check that $\mathcal{N}_{\mu}$ has weak covering $\omega_{1}$. Clearly $\operatorname{wcov}\left(\mathcal{N}_{\mu}\right) \geq \omega_{1}$.

Let $\left(B_{\alpha}\right)_{\alpha<\omega_{1}}$ be a family of Borel sets which is $\mu$-dense (with respect to symmetric difference). Denote by $X$ the set of points in $K$ which have countable character. For every $x \in X$ choose a countable base $\left(V_{n}(x)\right)_{n \in \omega}$ at $x$. Further, let $X_{\alpha}$ be the set of those $x \in X$ for which every $V_{n}(x)$ is approximated arbitrarily closely by the family $\left(B_{\beta}\right)_{\beta<\alpha}$. We have $X=$ $\bigcup_{\alpha<\omega_{1}} X_{\alpha}$; since $X$, by the assumption on $K$, meets every perfect set, it suffices to check that $\mu\left(X_{\alpha}\right)=0$ for every $\alpha<\omega_{1}$.

Suppose that $X_{\alpha}$ is of full outer measure for some $\alpha$ and let $\mathcal{A}$ be the algebra generated by $\left(B_{\beta}\right)_{\beta<\alpha}$. Consider an arbitrary open set $U$. For every $x \in Y=X_{\alpha} \cap U$ there is $n(x) \in \omega$ such that $V_{n(x)}(x) \subseteq U$. Writing $W=\bigcup_{x \in Y} V_{n(x)}(x)$ we have $Y \subseteq W \subseteq U$. It follows that $\mu(U \backslash W)=0$ and thus $U$ is approximated by $\mathcal{A}$. Consequently, $\mu$ is separable, which is a contradiction. An easy modification of this argument, taking into account the fact that $\mu$ is nowhere separable, gives $\mu\left(X_{\alpha}\right)=0$, and the proof is complete.

Let us note that Theorems 6.2 and 6.3 in fact mean that there is a nonseparable Radon measure for which $\operatorname{wcov}\left(\mathcal{N}_{\mu}\right)=\omega_{1}$ if and only if there is a nonseparable Radon measure on a compact space having a point of countable character in every perfect subset. We do not know whether the former condition is equivalent to $\operatorname{wcov}\left(\mathbb{L}_{\omega_{1}}\right)=\omega_{1}$. Recall that $\operatorname{cov}\left(\mathcal{N}_{\mu}\right)$, where $\mu$ is some Radon measure, is fully characterized by the properties of the measure algebra of $\mu$ (see 6.14(c) of [12]). The problem is if wcov has the same property, for instance, if $\operatorname{wcov}\left(\mathcal{N}_{\mu}\right)$ is constant for all homogeneous Radon measures $\mu$ of Maharam type $\omega_{1}$.

We end by showing how Martin's axiom affects weak coverings; see [11] for the terminology and notation concerning Martin's axiom. In particular, $\mathfrak{m}$ denotes the least cardinal $\kappa$ for which $\mathrm{MA}(\kappa)$ is false.

THEOREM 6.4. If $\mu$ is a nonatomic Radon measure then $\operatorname{wcov}\left(\mathcal{N}_{\mu}\right) \geq \mathfrak{m}$.

P r o o f. It suffices to consider a Radon measure $\mu$ on a compact space $K$. Given $\kappa<\mathfrak{m}$ and $\left(N_{\xi}\right)_{\xi<\kappa} \subseteq \mathcal{N}_{\mu}$, we are to find a perfect set in $K \backslash \bigcup_{\xi<\kappa} N_{\xi}$.

As $\mu$ is nonatomic we can find and fix a countable family $\mathcal{D}$ of closed subsets of $K$ of positive measure such that for every $F \in \mathcal{D}$ and $\varepsilon>0$ there 
are $n \in \omega$ and a pairwise disjoint family $\left(F_{i}\right)_{i \leq n} \subseteq \mathcal{D}$ such that every $F_{i}$ is contained in $F$ with $\mu\left(F_{i}\right)<\varepsilon$, and $\mu\left(F \backslash \bigcup_{i \leq n} F_{i}\right)<\varepsilon$.

We consider the set $\mathbf{P}$ of quadruples $(n, D, a, F)$, where:

(i) $n \in \omega$ and $D=\left(D_{s}\right)_{s \in 2<n}$ is a dyadic system of sets from $\mathcal{D}$;

(ii) $a$ is a finite subset of $\kappa$ and $F$ is a closed subset of $K \backslash \bigcup_{\xi \in a} N_{\xi}$;

(iii) $\mu\left(F \cap D_{s}\right)>0$ for every $s \in 2^{<n}$.

We declare $(n, D, a, F) \leq\left(n^{\prime}, D^{\prime}, a^{\prime}, F^{\prime}\right)$ if $n \leq n^{\prime}, D$ is extended by $D^{\prime}$, $a \subseteq a^{\prime}$ and $F \supseteq F^{\prime}$.

Consider a fixed $n$ and a dyadic system $D=\left(D_{s}\right)_{s \in 2<n}$. If $\mathcal{F}$ is an uncountable family of closed sets satisfying (iii) then there are sets $F_{k}$ 's $\in \mathcal{F}$ and $\delta>0$ such that $\mu\left(F_{k} \cap D_{s}\right) \geq \delta$ for every $s \in 2^{<n}$ and every $k$. It is easily seen that there are $i \neq j$ such that $\mu\left(F_{i} \cap F_{j} \cap D_{s}\right)>0$ for all $s$. This remark yields immediately that $\mathbf{P}$ is upwards ccc.

Given $k \in \omega$, the family $\{(n, D, a, F): n \geq k\}$ is cofinal in $\mathbf{P}$ (thanks to the way $\mathcal{D}$ is chosen). Moreover, for every $\xi<\kappa$, the family $\{(n, D, a, F)$ : $\xi \in a\}$ is easily seen to be cofinal in $\mathbf{P}$. Applying $\mathrm{MA}(\kappa)$ we find an upward directed $\mathbf{G}$ meeting the above families for every $k$ and $\xi$. Such a $\mathbf{G}$ brings forth a dyadic system $\left(D_{s}\right)_{s \in 2<\omega}$ and a closed set $F \subseteq K \backslash \bigcup_{\xi<\kappa} N_{\xi}$ such that $F \cap D_{s} \neq \emptyset$ for every $s \in 2^{<\omega}$. Thus, using Lemma 6.1 we infer that $F$ contains a perfect set, and the proof is complete.

Theorems 6.3 and 6.4 give immediately the following.

Corollary 6.5. Assume that $\mathfrak{m}>\omega_{1}$. If $X$ is a topological space such that for every compact perfect set $P \subseteq X$ there is $x \in P$ with $\chi(x, X)=\omega$ then every Radon measure on $X$ is separable.

Acknowledgements. I wish to thank Jacek Cichoń, Tasos Kamburelis and Janusz Pawlikowski for useful information. I also thank Professor Bogdan Węglorz for stimulating companionship during the 24th Winter School on Abstract Analysis (Czech Republic, January 96), when the main part of the research presented above was done.

N ot e. After the first version of this paper had been submitted for publication, David H. Fremlin showed that H $\left(\omega_{1}\right)$ is true assuming Martin's axiom and $\omega_{1}<\mathfrak{c}$, thus proving Haydon's conjecture. I am very grateful to D. Fremlin for sending me a preliminary note with his result and for his remarks concerning Lemma 2.4 .

\section{References}

[1] S. Argyros, S. Mercourakis and S. Negrepontis, Functional-analytic properties of Corson-compact spaces, Studia Math. 89 (1988), 197-229. 
[2] T. Bartoszyński and H. Judah, Set Theory: on the structure of the real line, A. K. Peters, 1995.

[3] T. Bartoszyński and S. Shelah, Closed measure zero sets, Ann. Pure Appl. Logic 58 (1992), 93-110.

[4] J. Brendle, H. Judah and S. Shelah, Combinatorial properties of Hechler forcing, Ann. Pure Appl. Logic 58 (1992), 185-199.

[5] J. Cichoń, A. Kamburelis and J. Pawlikowski, On dense subsets of the measure algebra, Proc. Amer. Math. Soc. 94 (1985), 142-146.

[6] J. Cichoń, A. Szymański and B. Węglorz, On intersections of sets of positive Lebesgue measure, Colloq. Math. 52 (1987), 173-177.

[7] W. W. Comfort and S. Negrepontis, Chain Conditions in Topology, Cambridge Univ. Press, 1982.

[8] R. G. Douglas, On extremal measures and subspace density, Michigan Math. J. 11 (1964), 243-246.

[9] M. Džamonja and K. Kunen, Measures on compact HS spaces, Fund. Math. 143 (1993), 41-54.

[10] - - - Properties of the class of measure separable compact spaces, ibid. 147 (1995), 261-277.

[11] D. H. Fremlin, Consequences of Martin's Axiom, Cambridge Univ. Press, Cambridge, 1984 .

[12] - Measure algebras, in: Handbook of Boolean Algebras, J. D. Monk (ed.), NorthHolland, 1989, Vol. 3, Chap. 22, 877-980.

[13] —, Real-valued measurable cardinals, in: Israel Math. Conf. Proc. 6, 1993, 961-1044.

[14] R. Haydon, On Banach spaces which contain $l^{1}(\tau)$ and types of measures on compact spaces, Israel J. Math. 28 (1977) 313-324.

[15] - On dual $L^{1}$-spaces and injective bidual Banach spaces, ibid. 31 (1978) 142-152.

[16] K. Kunen, Set Theory, Stud. Logic 102, North-Holland, 1980.

[17] -, A compact L-space under CH, Topology Appl. 12 (1981), 283-287.

[18] K. Kunen and J. van Mill, Measures on Corson compact spaces, Fund. Math. 147 (1995), 61-72.

[19] G. Plebanek, Compact spaces that result from adequate families of sets, Topology Appl. 65 (1995), 257-270.

[20] - On Radon measures on first-countable spaces, Fund. Math. 148 (1995), 159-164.

[21] Z. Semadeni, Banach Spaces of Continuous Functions, PWN, Warszawa, 1971.

[22] B. E. Shapirovskiŭ, Special types of embeddings in Tychonoff cubes. Subspaces of $\Sigma$-products and cardinal invariants, in: Á. Császár (ed.), Topology, Vol. II, NorthHoland, Amsterdam, 1980, 1055-1086.

[23] J. E. Vaughan, Small uncountable cardinals and topology, in: Open Problems in Topology, J. van Mill and G. M. Reed (eds.), North-Holland, 1990, Chapter 11, $195-216$.

Institute of Mathematics

Polish Academy of Sciences

Kopernika 18

51-617 Wrocław, Poland

E-mail: grzes@math.uni.wroc.pl

Received 7 May 1996;

in revised form 14 November 1996 and 30 January 1997 